\title{
Der Bregma-Test (BT) - Ein orientierender Test zur Abschätzung der Störungen der allgemeinen sensomotorischen Fähigkeiten im tiefen stabilisierenden System
}

\author{
The Bregma-Test (BT) - An Screening Test for Estimating the \\ Disturbances of General Senso-motoric Skills in the Deep \\ Stabilizing System
}

\author{
Autoren \\ Norman Best ${ }^{1,2}$, Stefanie Best ${ }^{1}$, Barbara Bocker ${ }^{1}$, Matthias Aurich ${ }^{2,3}$ \\ Institute \\ 1 Institut für Physiotherapie, Universitätsklinikum Jena, Jena \\ 2 Posture and Motion Group am Institut für Physiotherapie des UKJ \\ 3 Klinikum Ingolstadt, Zentrum für Orthopädie und Unfallchirurgie \\ Schlüsselwörter \\ Bewegungstherapie, Rückenschmerzen, sensomotorisches Training \\ Key words \\ exercise therapy, low back pain, sensoric motor training \\ eingereicht 12.01.2017 \\ akzeptiert 28.02.2017 \\ Bibliografie \\ DOI https://doi.org/10.1055/s-0043-104865 \\ Phys Med Rehab Kuror 2017; 27: 83-86 \\ (c) Georg Thieme Verlag KG Stuttgart · New York \\ ISSN 0940-6689 \\ Korrespondenzadresse \\ Dr. Norman Best \\ Institut für Physiotherapie \\ Universitätsklinikum Jena \\ Am Klinikum 1 \\ 07747 Jena \\ norman.best@gmx.de
}

\section{ZUSAMMENFASSUNG}

Hintergrund Das tiefe stabilisierende System arbeitet im Stand der Schwerkraft entgegen. Dabei ermöglicht ein funktionierendes System eine physiologische Haltung. Zur Evaluierung der posturalen Funktion werden einfache Screeningtests benötigt.

Beschreibung Nach manueller Stimulation (Fazilitation) am Bregma erfolgt die Aufforderung, dieses Richtung Decke zu bewegen und dabei die Füße auf dem Boden zu lassen.

Einschätzung Je nach Bewegung des Bregma unterscheiden wir in Grad 1 oder 2. Werden die Bregmabewegungen mit Parakinesen kombiniert, erfolgen Unterteilungen. Dadurch werden sensomotorische Störungen objektiv messbar.

Konsequenzen Bei deutlichem Störungsmuster ist eine initiale Körperwahrnehmungsschulung vor krankengymnastischen Konzepten anzustreben.

\section{ABSTRACT}

Background In an upright position the deep stabilizing system works against gravity, thereby allowing a physiological posture. Simple screening tests are needed to evaluate the postural function.

Description Following a manual stimulation (facillitation) of the Bregma, the patient will be asked to move the Bregma towards the ceiling while leaving both feet on the ground.

Assessment Depending on the movement of the Bregma, we distinguish grade 1 or 2 . Further subdivisions are made if the movements of the Bregma are combined with parakinetic activities. Sensomotor disorders are thus measured objectively.

Consequences In the case of a clear disorder pattern, an initial body awareness training must be applied before physical exercise programs are started.

\section{Hintergrund}

Das tiefe stabilisierende System arbeitet der Schwerkraft entgegen und „im Stillen“. Im Rahmen diverser Stereotype sind dabei stabilisierende Aufgaben zu erfüllen. Einer der Stereotype ist das Stehen. Aufrechtes Stehen ist keine statische Aufgabe sondern ein dynamischer Prozess des verhinderten Umfallens, wie Ekkard Geipel einmal treffend formulierte [1].

Subkortikal gespeicherte Bewegungsabläufe garantieren die mehr oder weniger aufrechte Position des "Homo erectus", die uns das Überleben vor Urzeiten in der Steppe ermöglicht hat, da dadurch eine bessere Übersicht im hohen Gras zu erreichen war [2].
Allerdings ist dieses quasi autonom arbeitende System auf ausreichenden positiven Input angewiesen. In der modernen Zivilgesellschaft zeigt sich, dass dieser Input, den man einfach mit „allgemeiner Bewegung “ umschreiben kann, fehlt. Es ist in weiten Teilen der Bevölkerung bekannt, dass Bewegung „gesund“ ist. Dennoch ist der übliche Alltag von Verharren in Zwangshaltungen, sitzender Tätigkeit oder aber, im anderen Extrem, von Überlastungsbewegungen geprägt.

Somit verkümmert das tiefe stabilisierende System oder es müssen sich Kompensationsmechanismen herausbilden, die mitunter zu Beschwerden im Bewegungssystem führen und darüber hinaus die gesamte Körperlichkeit negativ beeinflussen können. 
Der Idee für die Entwicklung und Beschreibung dieses Tests rührt daher, dass in der klinischen Praxis schnelle und einfache Suchtests- bzw. Screeningtest benötigt werden, die keinen Aufwand wie in einem Labortest, aber dennoch eine gute Aussagekraft besitzen. Diese Tests können in der Regel nur beschreibenden Charakter haben und dem Untersucher eine Orientierung im weiteren klinischen Handling mit dem Patienten bieten. Ein schneller und einfacher Test zur Einschätzung der sensomotorischen Ansteuerung im klinischen Alltag lag beim untersuchten Patientenklientel des Autors nahe.

\section{Das Bregma}

Das Bregma ist der Punkt, der aus der Pfeilnaht (Sutura sagittalia) und der Kranznaht (Sutura coronaria) gebildet wird. Die ersten Lebensjahre ist die Schädeldecke an dieser Stelle geöffnet und wird vordere Fontanelle genannt [3] (

\section{Das tiefe stabilisierende System}

Das tiefe stabilisierende System (TSS) wurde unter anderem von Karel Lewit beschrieben. Es sichert die aufrechte Körperhaltung des Menschen (Homo erectus) gegen die Schwerkraft. Neben den tiefen kleinen Rückenmuskeln (Mm. multifidii) und dem M. transver-

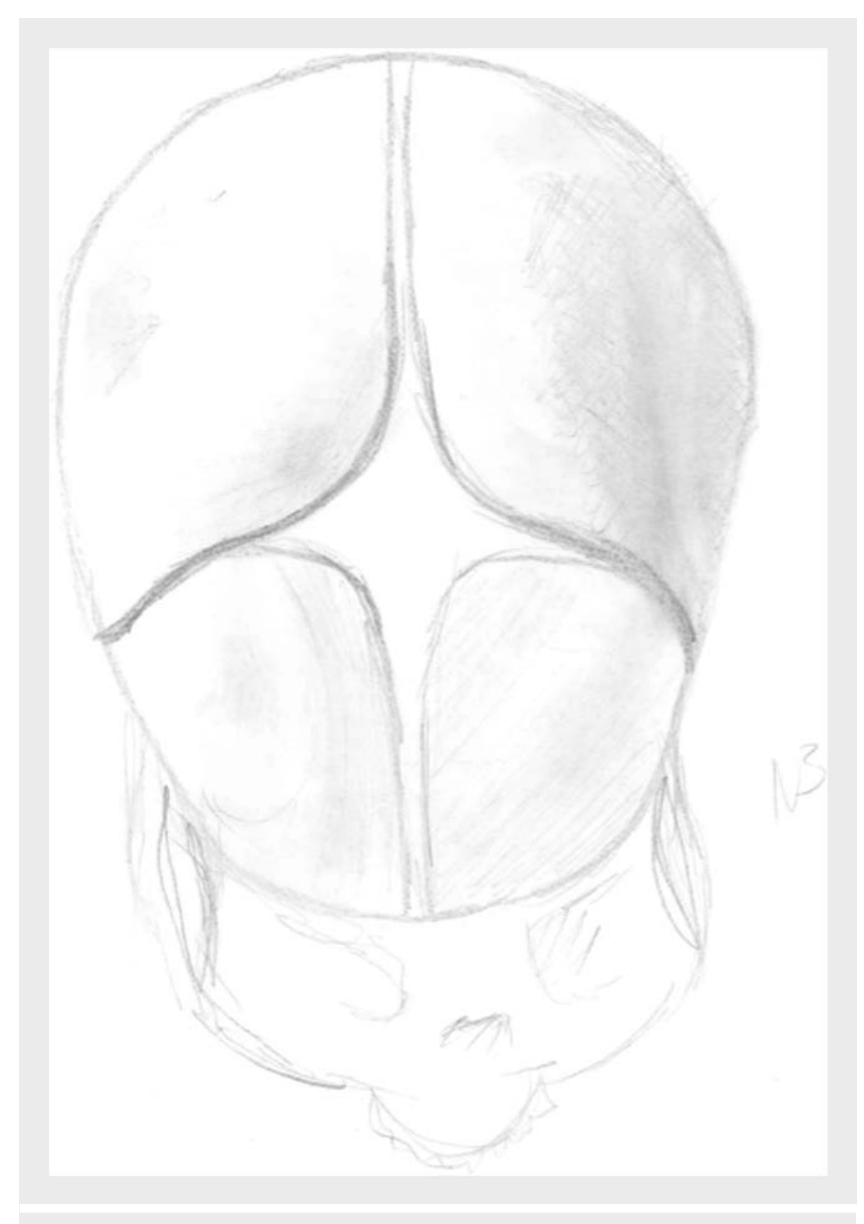

- Abb. 1 Bregma beim Kind (vordere Fontanelle); Grafik: Nelly Best. sus abdominis werden der M. quadratus plantae, die Pars aszendens des M. trapezius und der M. serratus anterior sowie die tiefen Halsbeuger zum TSS gezählt. Des Weiteren werden aber ebenfalls das Zwerchfell und das Beckenbodensystem hinzugerechnet. Ohne deren gute Funktionsfähigkeit ist eine ökonomische aufrechte Körperhaltung nicht möglich [4] .

\section{Bregma-Test}

\section{Ablauf}

Der Patient steht in seinem normalen „Muster“, der Untersucher ca. $45^{\circ}$ schräg davor. Der Untersucher berührt das Bregma und fazilitiert diesen Punkt durch leichte „Kratzbewegungen“. Durch diese Fazilitation soll erreicht werden, dass dieser Punkt, bzw. das rezeptive Feld dieses Punktes in einen erhöhten Wahrnehmungsmodus gebracht wird, d. h. dieser Punkt „bewusst gemacht“ wird. Der Kontakt zum Bregma wird nun aufgegeben und es folgt folgendes Kommando: „Bitte diesen Punkt in Richtung Decke bewegen ohne die Fersen abzuheben." Nun soll also der Patient die Kalotte Richtung Decke bewegen, und zwar so, dass sich das Bregma Richtung Decke bewegt. Es wird beobachtet, ob dies gelingt und wie der Patient die dazu notwendigen Bewegungen ausführt ( Abb. 3).

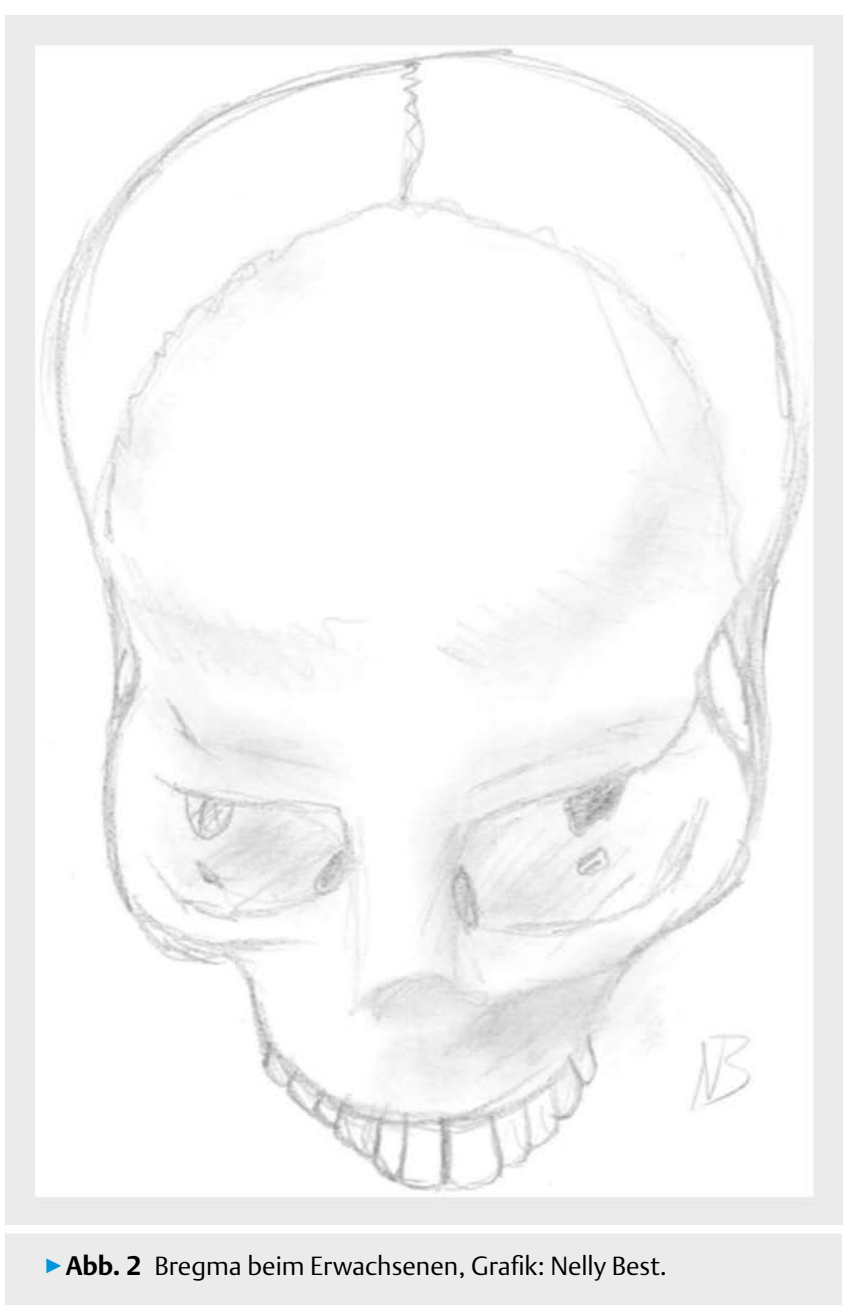




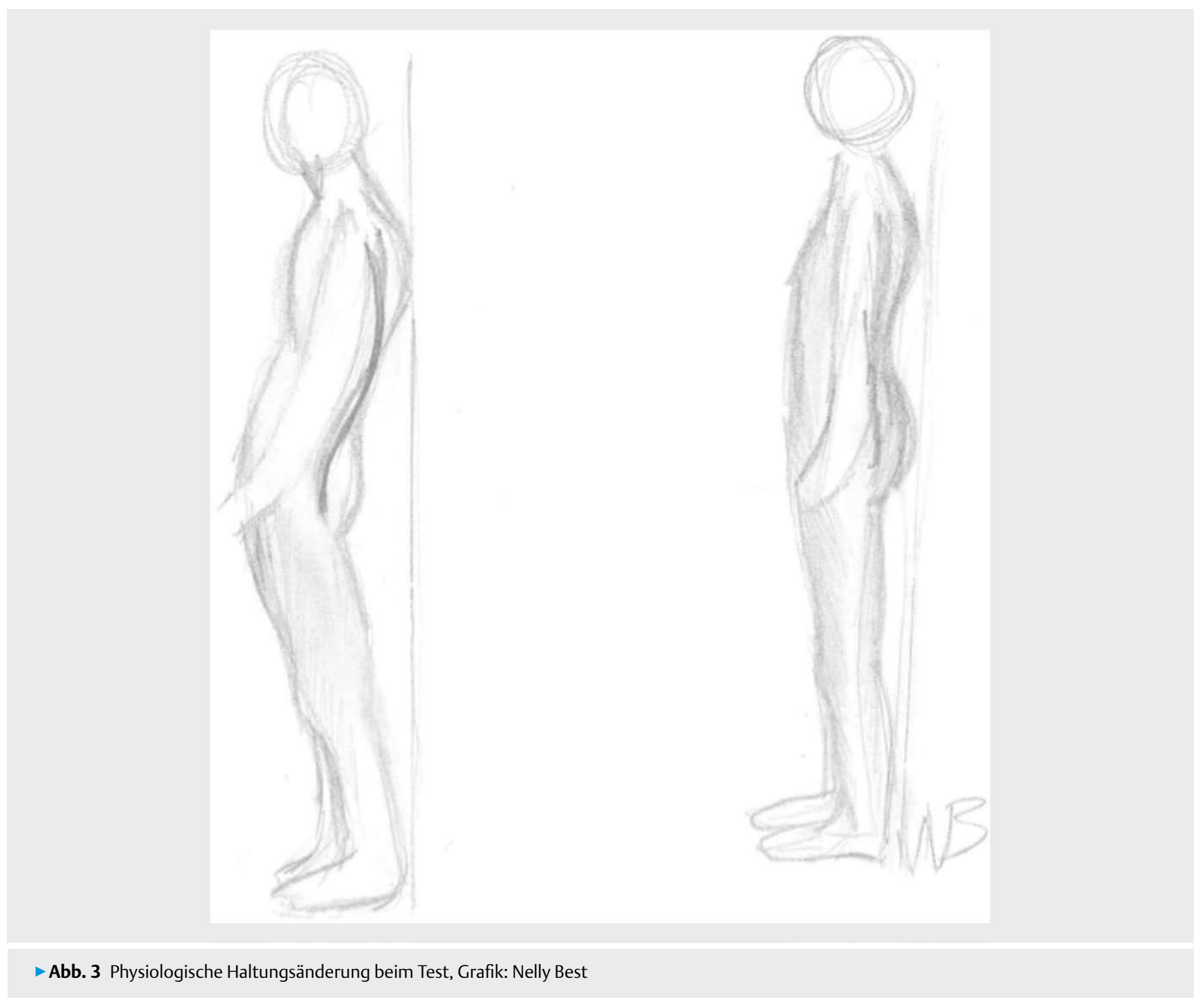

\section{Auswertung}

Die zu erwartenden Bewegungen sollten im Idealfall die Schädelkalotte direkt Richtung Decke bringen und damit zu einer Vertikalisierung des Bregma führen. Das kann nur gelingen, wenn sich die Schwünge der Wirbelsäule in allen Bereichen dem Kopflot annähern. Dabei werden die Schultergelenke retrahiert und das Becken aufgerichtet. Die Kyphose der BWS vermindert sich, genauso wie die Lordose der LWS. Es ist anzunehmen, dass das Os sacrum eine Gegennutationsbewegung ausführen muss, um die Beckenaufrichtung zu unterstützen. Erfolgen diese „Korrekturen der Haltung“ ohne Parakinesen sprechen wir vom Grad 1.0 des BT. Es gibt bei dieser Ausführung keinen Hinweis auf sensomotorische Defizite.

In der Betreuung von Patienten fiel des Öfteren auf, dass die Bregmabewegung korrekt, allerdings mit Nebenbewegungen ausgeführt wurde, bei der Nutzung der Schultergürtelheber (M. trapezius Pars deszendens, M. levator scapulae) oft auch mit einer Einatmungsinitiierung. Bei dieser Ausführung sprechen wir vom Grad 1.1. Sollten orofaziale Nebenbewegungen auftreten ist das Ergebnis des BT Grad 1.2. In der Kombination beider Parakinesen ergibt der Test als Ergebnis Grad 1.3.
Grad 1.0: Ausführung korrekt, keine Parakinesen

Grad 1.1: Bregma wird korrekt angehoben, Schultern werden ebenfalls mit angehoben

Grad 1.2: Bregma wird korrekt angehoben, faziale Muskulatur wird mit angespannt

Grad 1.3: Bregma wird korrekt angehoben, Schultergürtel und orofaziales System wird mit bewegt

Die untersuchten Menschen, die nicht in der Lage sind willentlich die Muskulatur so anzuspannen, dass das Bregma eine Vertikalisierung ausführt, sondern der Kopf frontal angehoben wird und somit das Bregma eher nach hinten eine Kreisbahn beschreibt erhalten Grad 2.0. Entsprechend der Einteilung bei Grad 1 ergeben sich Abstufungen im Sinne eines Grad 2.1 für parakinetische Aktivierung des Schultergürtels bzw. Grad 2.2 für eine orofaziale Nebenbewegung und Grad 2.3 für die Kombination aus Grad 2.1 und 2.2

Grad 2.0 Kopf neigt sich nach hinten, Stirn wird angehoben, Bregma sinkt nach hinten ab

Grad 2.1: Grad 2 in Kombination mit Schulterheben

Grad 2.2: Grad 2 in Kombination mit Irritation der Gesichtsmuskulatur

Grad 2.3: Kombination aus Grad 2.1 und 2.2 ( $>$ Abb. 4) 


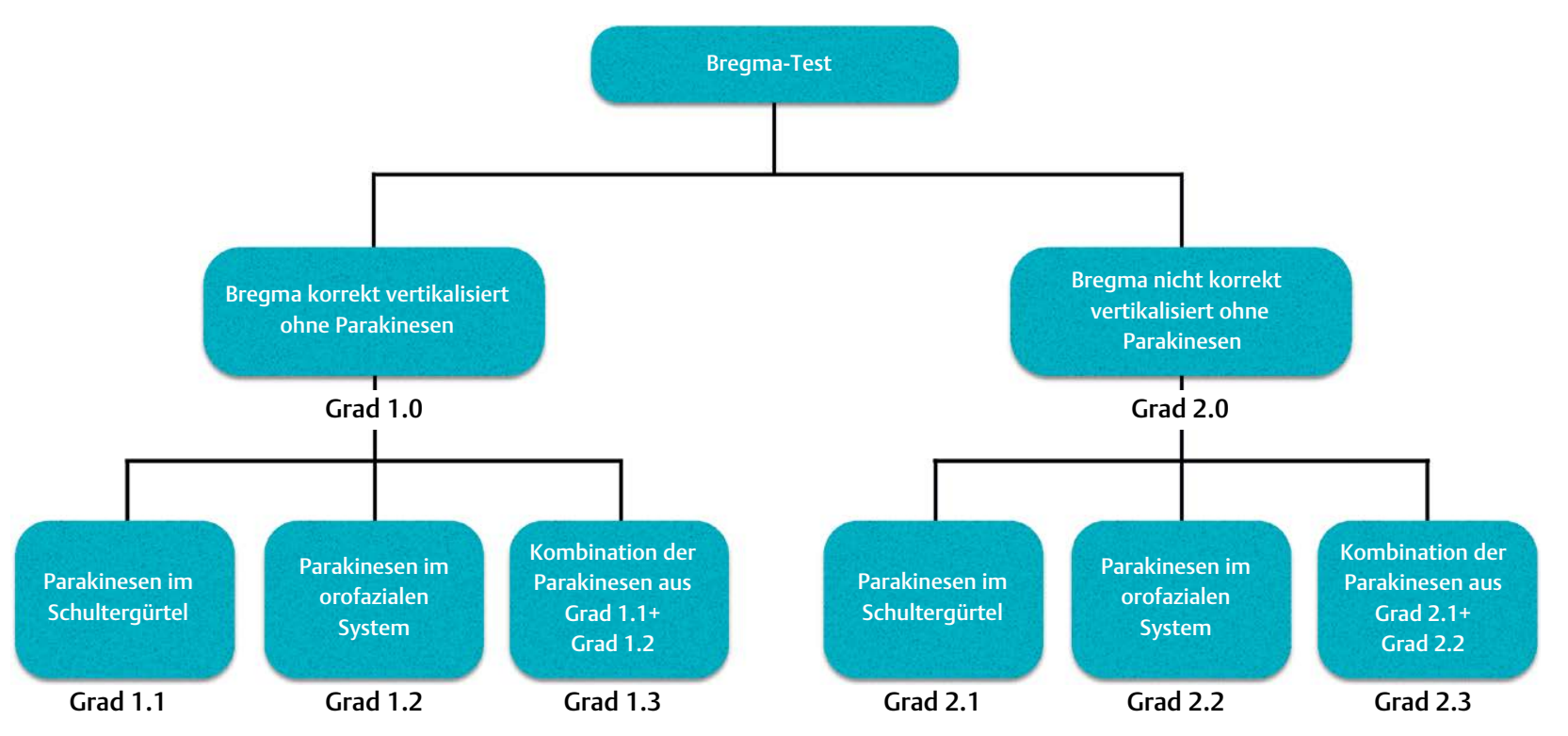

Abb. 4 Graduierungsschema.

\section{Konsequenzen}

Durch die dargebotenen Bewegungsabläufe lassen sich nun Rückschlüsse auf die Fähigkeit, die statiksichernde Muskulatur anzusteuern, schließen. Der Untersucher kann daraus ableiten, ob der Patient willentlich Muskelgruppen so ansteuern kann, dass eine vom Arzt vorgegebene Änderung der Haltung (des Standstereotyps) zumindest kurzzeitig erfolgen kann. Aus Sicht des Verfassers lässt dies Rückschlüsse hinsichtlich des weiteren Vorgehens in der körperzentrierten Arbeit mit dem Patienten zu. Wird der Test physiologisch ausgeführt, können Einheiten medizinischer Trainingstherapie und gerätegestützter Krankengymnastik erfolgen, die darauf abzielen eine stabilisierende Wirkung zu entfalten. Dabei zeigte ich bei der empirischen Betrachtung von Einzelfällen, dass die motorische Ansteuerung bei den auszuführenden Bewegungen auch innerhalb der physiologischen Gruppe noch deutlich variieren kann. Insgesamt scheint es aber genau die Gruppe zu sein, die das Potential eines Gerätetrainings im rehabilitativen Sinne voll ausschöpfen kann, wie z. B. Übungen auf bzw. mit dem Posturomed oder Propriomed, Seilzugsysteme sowie koordinative Übungen auf instabilen Unterlagen.

Diejenigen Patienten die nicht in der Lage sind das Bregma kontrolliert Richtung Decke zu verschieben, sollten aus Autorensicht initial eine Körperwahrnehmungsschulung und einem propriozeptiven Trainings zugeführt werden. Dies liegt in der Tatsache begründet, dass die Menschen mit sensomotorischen Schwierigkeiten, die Ihnen abverlangten Übungen, welche eine hohe Geschicklichkeit im sensomotorischen Bereich verlangen, gar nicht korrekt ausführen können, sondern beim Training dauerhaft in Überlastungssituationen kommen oder aber kompensatorische Fehlstereotype beanspruchen und somit das Ziel der Intervention verfehlen. Es zeigt sich in Beobachtungen, dass die Körperwahrnehmungsarbeit schnell eine Besserung bringt und die betroffenen Patienten dann beginnen können, die eigentlich vorgesehenen Übungen absolvieren zu können.

Der BT kann im Verlauf sehr einfach zu Verlaufskontrollen dienen und den Zeitpunkt bestimmen helfen, zu dem die sensomotorisch anspruchsvolleren Übungen eingebaut werden können.

\section{Schlussfolgerungen}

In der täglichen Routine scheint der BT einfach und schnell in der Durchführung. Nach der inhaltlichen Beschreibung muss nun die statistische Bearbeitung hinsichtlich der Testgütekriterien erfolgen. Initial sind die Hauptkriterien zuerst zu erfassen [5].

\section{Interessenkonflikt}

Die Autoren geben an, dass kein Interessenkonflikt besteht.

\section{Literatur}

[1] Geipel E. 2013; Seminar zur Untersuchung und Einschätzung des Standstereotyps im Rahmen des Kursleiterkurses der ÄMM.

[2] Bruggencate ten G, Dieringer N. Sensomotorische Systeme: Körperhaltung, Bewegung und Blickmotorik. In: Klinke R, Silbernagel S, (Hrsg.). Lehrbuch der Physiologie. 4., korrigierte Aufl. Stuttgart: Thieme; 2003: S 653-S 700

[3] Lang J. Schädel, Übersicht. In: Lang J, (Hrsg.). Klinische Anatomie des Kopfes. Berlin: Springer; 1981: S 2-22

[4] Lewit K, Kobesova A, Lepšíková M. Das tiefe stabilisierende System der Wirbelsäule - Seine Bedeutung für funktionelles Denken. Manuelle Medizin 2010; 48: 440

[5] Lienert GA, Raatz U. Testaufbau und Testanalyse. 6. Aufl. Weinheim: Beltz; 1998 


\section{Hinweis}

Dieser Artikel wurde gemäß des Erratums vom 11.7.2017 geändert.

\section{Erratum}

Im Beitrag „Der Bregma-Test (BT) - Ein orientierender Test zur Abschätzung der Störungen der allgemeinen sensomotorischen Fähigkeiten im tiefen stabilisierenden System“, Norman Best, Stefanie Best, Barbara Bocker, Matthias Aurich, Phys Med Rehab Kuror 2017; 27: 83-86 (DOI 10.1055/s-0043-104865) hat sich leider in der Abbildung 4 (Graduierungsschema ein Fehler eingeschlichen.

Die Abbildung wurde ersetzt. 